\title{
Some identities involving Gegenbauer polynomials
}

Dae San Kim', Taekyun Kim²* and Seog-Hoon Rim

"Correspondence: tkkim@kw.ac.kr ${ }^{2}$ Department of Mathematics, Kwangwoon University, Seoul, 139-701, Republic of Korea Full list of author information is available at the end of the article

\section{Abstract \\ In this paper, we derive some interesting identities involving Gegenbauer polynomials arising from the orthogonality of Gegenbauer polynomials for the inner product space $\mathbb{P}_{n}$ with respect to the weighted inner product $\left\langle p_{1}, p_{2}\right\rangle=\int_{-1}^{1} p_{1}(x) p_{2}(x)\left(1-x^{2}\right)^{\lambda-\frac{1}{2}} d x$}

\section{Introduction}

The Gegenbauer polynomials are given in terms of the Jacobi polynomials $P_{n}^{(\alpha, \beta)}(x)$ with $\alpha=\beta=\lambda-\frac{1}{2}\left(\lambda>-\frac{1}{2}, \lambda \neq 0\right)$ by

$$
\begin{aligned}
C_{n}^{(\lambda)}(x) & =\frac{\Gamma\left(\lambda+\frac{1}{2}\right) \Gamma(n+2 \lambda)}{\Gamma(2 \lambda) \Gamma\left(n+\lambda+\frac{1}{2}\right)} P_{n}^{\left(\lambda-\frac{1}{2}, \lambda-\frac{1}{2}\right)}(x) \\
& =\left(\begin{array}{c}
n+2 \lambda-1 \\
n
\end{array}\right) \sum_{k=0}^{n} \frac{\left(\begin{array}{l}
n \\
k
\end{array}\right)(2 \lambda+n)_{k}}{\left(\lambda+\frac{1}{2}\right)_{k}}\left(\frac{x-1}{2}\right)^{k},
\end{aligned}
$$

where $(a)_{k}=a(a+1)(a+2) \cdots(a+k-1)($ see $[1,2])$.

From (1.1), we note that $C_{k}^{(\lambda)}(x)$ is a polynomial of degree $n$ with real coefficients and $C_{n}^{(\lambda)}(1)=\left(\begin{array}{c}n+2 \lambda-1 \\ n\end{array}\right)$. The leading coefficient of $C_{n}^{(\lambda)}(x)$ is $2^{n}\left(\begin{array}{c}\lambda+n-1 \\ n\end{array}\right)$. By the theory of Jacobi polynomials with $\alpha=\beta=\lambda-\frac{1}{2}, \lambda>-\frac{1}{2}$, and $\lambda \neq 0$, we get

$$
C_{n}^{(\lambda)}(-x)=(-1)^{n} C_{n}^{(\lambda)}(x)
$$

It is not difficult to show that $C_{n}^{(\lambda)}(x)$ is a solution of the following Gegenbauer differential equation:

$$
\left(1-x^{2}\right) y^{\prime \prime}-(2 \lambda+1) x y^{\prime}+n(n+2 \lambda) y=0
$$

The Rodrigues formula for the Gegenbauer polynomials is well known as the following:

$$
\left(1-x^{2}\right)^{\lambda-\frac{1}{2}} C_{n}^{(\lambda)}(x)=\frac{(-2)^{n}(\lambda)_{n}}{n !(n+2 \lambda)_{n}}\left(\frac{d}{d x}\right)^{n}\left(1-x^{2}\right)^{n+\lambda-\frac{1}{2}} \quad(\text { see }[3,4])
$$

Equation (1.3) can be easily derived from the properties of Jacobi polynomials.

\section{Springer}

(c) 2012 Kim et al.; licensee Springer. This is an Open Access article distributed under the terms of the Creative Commons Attribution License (http://creativecommons.org/licenses/by/2.0), which permits unrestricted use, distribution, and reproduction in any medium, provided the original work is properly cited. 
As is well known, the generating function of Gegenbauer polynomials is given by

$$
\frac{2^{\lambda-\frac{1}{2}}}{\left(1-2 x t+t^{2}\right)^{\frac{1}{2}}\left(1-x t+\sqrt{1-2 x t+t^{2}}\right)^{\lambda-\frac{1}{2}}}=\sum_{n=0}^{\infty} \frac{\left(\lambda+\frac{1}{2}\right)_{n}}{(2 \lambda)_{n}} C_{n}^{(\lambda)}(x) t^{n} .
$$

Equation (1.4) can be also derived from the generating function of Jacobi polynomials.

From (1.4), we note that

$$
\frac{1}{\left(1-2 x t+t^{2}\right)^{\lambda}}=\sum_{n=0}^{\infty} C_{n}^{(\lambda)}(x) t^{n} \quad(|t|<1,|x| \leq 1)
$$

The proof of (1.5) is given in the following book: Stein and Weiss, Introduction to Fourier Analysis in Euclidean Space, Princeton University Press, 1971.

By (1.1) and (1.2), we get

$$
\int_{-1}^{1} C_{n}^{(\lambda)}(x) C_{m}^{(\lambda)}(x)\left(1-x^{2}\right)^{\lambda-\frac{1}{2}} d x=\frac{\pi 2^{1-2 \lambda} \Gamma(n+2 \lambda)}{n !(n+\lambda)(\Gamma(\lambda))^{2}} \delta_{m, n}
$$

where $\delta_{m, n}$ is the Kronecker symbol and it holds for each fixed $\lambda \in \mathbb{R}$ with $\lambda>-\frac{1}{2}$ and $\lambda \neq 0$.

Equation (1.6) implies the orthogonality of $C_{n}^{(\lambda)}(x)$ and equation (1.6) is important in deriving our results in this paper. From (1.5), we can derive the following derivative of Gehenbauer polynomials $C_{n}^{(\lambda)}(x)$ :

$$
\frac{d}{d x} C_{n}^{(\lambda)}(x)=2 \lambda C_{n-1}^{(\lambda+1)}(x), \quad \text { for } n \geq 1
$$

By (1.7), we get

$$
\frac{d^{k}}{d x^{k}} C_{n}^{(\lambda)}(x)=2^{k} \lambda^{k} C_{n-k}^{(\lambda+k)}(x)
$$

As is well known, the Bernoulli polynomials $B_{n}(x)$ are defined by the generating function to be

$$
\frac{t}{e^{t}-1} e^{x t}=e^{B(x) t}=\sum_{n=0}^{\infty} B_{n}(x) \frac{t^{n}}{n !} \quad(\text { see }[5-13])
$$

with the usual convention about replacing $B^{n}(x)$ by $B_{n}(x)$. In the special case, $x=0, B_{n}(0)=$ $B_{n}$ are called the $n$th Bernoulli numbers.

From (1.9), we note that

$$
B_{n}(x)=(B+x)^{n}=\sum_{l=0}^{n}\left(\begin{array}{l}
n \\
l
\end{array}\right) B_{n-l} x^{l} \quad(\text { see }[9-13]),
$$

and

$$
B_{n}^{\prime}(x)=\frac{d}{d x} B_{n}(x)=n B_{n-1}(x) .
$$


The Euler polynomials $E_{n}(x)$ are also defined by the generating function to be

$$
\frac{2}{e^{t}+1} e^{x t}=e^{E(x) t}=\sum_{n=0}^{\infty} E_{n}(x) \frac{t^{n}}{n !} \quad(\text { see }[4,14-18])
$$

with the usual convention about replacing $E^{n}(x)$ by $E_{n}(x)$. In the special case, $x=0, E_{n}(0)=$ $E_{n}$ are called the $n$th Euler numbers. By (1.12), we see that the recurrence formula for $E_{n}$ is given by

$$
E_{0}=1, \quad(E+1)^{n}+E_{n}=2 \delta_{0, n} \quad(\text { see }[19-21]) .
$$

For each fixed $\lambda \in \mathbb{R}$ with $\lambda>-\frac{1}{2}$ and $\lambda \neq 0$, let $\mathbb{P}_{n}=\{p(x) \in \mathbb{R}[x] \mid \operatorname{deg} p(x) \leq n\}$ be an inner product space with respect to the inner product

$$
\left\langle p_{1}(x), p_{2}(x)\right\rangle=\int_{-1}^{1}\left(1-x^{2}\right)^{\lambda-\frac{1}{2}} p_{1}(x) p_{2}(x) d x
$$

where $p_{1}(x), p_{2}(x) \in \mathbb{P}_{n}$. The information entropy of Gegenbauer polynomials is relevant since it is related to the angular part of the information entropies of certain quantum mechanical systems such as the harmonic oscillator and the hydrogen atom in $D$ dimensions. In [7], Buyarov, Lopez-Artes, Martinez-Finkelshtein, and Van Assche gave an effective method to compute the entropy for Gegenbauer polynomials with an integer parameter and obtain the first few terms in the asymptotic expansion as the degree of the polynomial tends to infinity. That is, an efficient method was provided for evaluating, in a closed form, the information entropy of the Gegenbauer polynomials $C_{n}^{(\lambda)}(x)$ in the case when $\lambda=l \in \mathbb{N}$. For given values of $n$ and $l$, this method requires the computation by means of recurrence relations of two auxiliary polynomials, $P(x)$ and $H(x)$, of degrees $2 l-2$ and $2 l-4$, respectively (see [18]). In [18], Sanchez-Ruiz showed that $P(x)$ is related to the coefficients of the Gaussian quadrature formula for the Gegenbauer weights $w_{l}(x)=\left(1-x^{2}\right)^{l-\frac{1}{2}}$, and this fact is used to obtain the explicit expression of $P(x)$. The position and momentum information entropies of $D$-dimensional quantum systems with central potentials, such as the isotropic harmonic oscillator and the hydrogen atom, depend on the entropies of the (hyper)spherical harmonics (see [2]). In turn, these entropies are expressed in terms of the entropies of the Gegenbauer (ultraspherical) polynomials $C_{n}^{(\lambda)}(x)$, the parameter $\lambda$ being either an integer or a half-integer number. Up to now, however, the exact analytical expression of the entropy of Gegenbauer polynomials of arbitrary degree $n$ has only been obtained for the particular values of the parameter $\lambda=0,1,2$ (see [2]). In [2], de Vicente, Gandy, Sanchez-Ruiz presented a novel approach to the evaluation of the information entropy of Gegenbauer polynomials, which makes use of trigonometric representations for these polynomials and complex integration techniques. Using this method, we are able to find the analytical expression of the entropy for arbitrary values of both $n$ and $\lambda \in \mathbb{N}$ (see [2]). The Gegenbauer polynomial seems to be interesting and important in the area of mathematical physics. Recently, many authors have studied Gegenbauer polynomials related to mathematical physics (see $[1-5,7,10,11,14,18,21,22])$. In this paper, we derive some interesting identities involving Gegenbauer polynomials arising from the orthogonality of those for the inner product space $\mathbb{P}_{n}$ with respect to the weighted inner product $\left\langle p_{1}, p_{2}\right\rangle=\int_{-1}^{1} p_{1}(x) p_{2}(x)\left(1-x^{2}\right)^{\lambda-\frac{1}{2}} d x$. 
Our methods used in this paper are useful in finding some new identities and relations on the Bernoulli and Euler polynomials involving Gegenbauer polynomials.

\section{Some identities involving Gegenbauer polynomials}

Let us take $p(x)=\sum_{k=0}^{n} d_{k} C_{k}^{(\lambda)}(x) \in \mathbb{P}_{n}, d_{k} \in \mathbb{R}$. Then, by (1.6) and (1.14), we get

$$
\begin{aligned}
\left\langle p(x), C_{k}^{(\lambda)}(x)\right\rangle & =d_{k}\left\langle C_{k}^{(\lambda)}(x), C_{k}^{(\lambda)}(x)\right\rangle \\
& =d_{k} \int_{-1}^{1}\left(1-x^{2}\right)^{\lambda-\frac{1}{2}} C_{k}^{(\lambda)}(x) C_{k}^{(\lambda)}(x) d x=d_{k} \frac{\pi 2^{1-2 \lambda} \Gamma(k+2 \lambda)}{k !(k+\lambda)(\Gamma(\lambda))^{2}} .
\end{aligned}
$$

Thus, from (2.1), we have

$$
d_{k}=\frac{(\Gamma(\lambda))^{2} k !(k+\lambda)}{\pi 2^{1-2 \lambda} \Gamma(k+2 \lambda)} \int_{-1}^{1}\left(1-x^{2}\right)^{\lambda-\frac{1}{2}} p(x) C_{k}^{(\lambda)}(x) d x .
$$

By (1.3) and (2.2), we get

$$
\begin{aligned}
d_{k} & =\frac{(\Gamma(\lambda))^{2} k !(k+\lambda)}{\pi 2^{1-2 \lambda} \Gamma(k+2 \lambda)} \times \frac{(-2)^{k}(\lambda)_{k}}{k !(k+2 \lambda)_{k}} \int_{-1}^{1}\left(\frac{d^{k}}{d x^{k}}\left(1-x^{2}\right)^{k+\lambda-\frac{1}{2}}\right) p(x) d x \\
& =\frac{(k+\lambda) \Gamma(\lambda)}{(-2)^{k} \sqrt{\pi} \Gamma\left(k+\lambda+\frac{1}{2}\right)} \int_{-1}^{1}\left(\frac{d^{k}}{d x^{k}}\left(1-x^{2}\right)^{k+\lambda-\frac{1}{2}}\right) p(x) d x
\end{aligned}
$$

Therefore, by (2.3), we obtain the following proposition.

Proposition 2.1 For $p(x) \in \mathbb{P}_{n}$, let

$$
p(x)=\sum_{n=0}^{n} d_{k} C_{k}^{(\lambda)}(x) \quad\left(d_{k} \in \mathbb{R}\right) .
$$

Then

$$
d_{k}=\frac{(k+\lambda) \Gamma(\lambda)}{(-2)^{k} \sqrt{\pi} \Gamma\left(k+\lambda+\frac{1}{2}\right)} \int_{-1}^{1}\left(\frac{d^{k}}{d x^{k}}\left(1-x^{2}\right)^{k+\lambda-\frac{1}{2}}\right) p(x) d x .
$$

For example, let $p(x)=x^{n} \in \mathbb{P}_{n}$. From Proposition 2.1, we note that

$$
\begin{aligned}
d_{k} & =\frac{(k+\lambda) \Gamma(\lambda)}{(-2)^{k} \sqrt{\pi} \Gamma\left(k+\lambda+\frac{1}{2}\right)} \int_{-1}^{1}\left(\frac{d^{k}}{d x^{k}}\left(1-x^{2}\right)^{k+\lambda-\frac{1}{2}}\right) x^{n} d x \\
& =(-n) \int_{-1}^{1}\left(\frac{d^{k-1}}{d x^{k-1}}\left(1-x^{2}\right)^{k+\lambda-\frac{1}{2}}\right) x^{n-1} d x \times\left(\frac{(k+\lambda) \Gamma(\lambda)}{\sqrt{\pi}(-2)^{k} \Gamma\left(k+\lambda+\frac{1}{2}\right)}\right) \\
& =\cdots \\
& =\frac{(k+\lambda) n ! \Gamma(\lambda)}{(n-k) ! 2^{k} \sqrt{\pi}\left(k+\frac{1}{2}+\lambda\right)} \int_{-1}^{1}\left(1-x^{2}\right)^{k+\lambda-\frac{1}{2}} x^{n-k} d x \\
& =\left(1+(-1)^{n-k}\right) \frac{(k+\lambda) n ! \Gamma(\lambda)}{(n-k) ! 2^{k} \sqrt{\pi} \Gamma\left(k+\frac{1}{2}+\lambda\right)} \int_{0}^{1}\left(1-x^{2}\right)^{k+\lambda-\frac{1}{2}} x^{n-k} d x .
\end{aligned}
$$


Let us assume that $n-k \equiv 0(\bmod 2)$. Then, by $(2.4)$, we get

$$
\begin{aligned}
d_{k} & =\frac{(k+\lambda) n ! \Gamma(\lambda)}{(n-k) ! 2^{k} \sqrt{\pi} \Gamma\left(k+\frac{1}{2}+\lambda\right)} B\left(k+\lambda+\frac{1}{2}, \frac{n-k+1}{2}\right) \\
& =\frac{\Gamma\left(\frac{n-k+1}{2}\right) \Gamma\left(k+\lambda+\frac{1}{2}\right)}{\Gamma\left(\frac{n+k+2 \lambda+2}{2}\right)}
\end{aligned}
$$

where $B(\alpha, \beta)$ is the beta function which is defined by $B(\alpha, \beta)=\frac{\Gamma(\alpha) \Gamma(\beta)}{\Gamma(\alpha+\beta)}$.

It is easy to show that

$$
\begin{aligned}
\Gamma\left(\frac{n-k+1}{2}\right) & =\frac{n-k-1}{2} \Gamma\left(\frac{n-k-1}{2}\right) \\
& =\left(\frac{n-k-1}{2}\right)\left(\frac{n-k-3}{2}\right) \Gamma\left(\frac{n-k-3}{2}\right)=\cdots \\
& =\frac{\left(\frac{n-k}{2}\right)\left(\frac{n-k-1}{2}\right)\left(\frac{n-k-2}{2}\right) \cdots \frac{2}{2} \Gamma\left(\frac{1}{2}\right)}{\left(\frac{n-k}{2}\right)\left(\frac{n-k-2}{2}\right) \cdots\left(\frac{2}{2}\right)} \\
& =\frac{(n-k) ! \sqrt{\pi}}{2^{n-k}\left(\frac{n-k}{2}\right) !} .
\end{aligned}
$$

Therefore, by (2.5) and (2.6), we obtain the following identity:

$$
x^{n}=\sum_{0 \leq k \leq n, n-k \equiv 0(\bmod 2)} \frac{(k+\lambda) n ! \Gamma(\lambda)}{2^{n}\left(\frac{n-k}{2}\right) ! \Gamma\left(\frac{n+k+2 \lambda+2}{2}\right)} C_{k}^{(\lambda)}(x) .
$$

Let us take $p(x)=B_{n}(x) \in \mathbb{P}_{n}$. Then, by (1.10), we get

$$
\begin{aligned}
d_{k} & =\frac{(k+\lambda) \Gamma(\lambda)}{(-2)^{k} \sqrt{\pi} \Gamma\left(k+\lambda+\frac{1}{2}\right)} \int_{-1}^{1}\left(\frac{d^{k}}{d x^{k}}\left(1-x^{2}\right)^{k+\lambda-\frac{1}{2}}\right) B_{n}(x) d x \\
& =\frac{(k+\lambda) \Gamma(\lambda)(-n)}{(-2)^{k} \sqrt{\pi} \Gamma\left(k+\lambda+\frac{1}{2}\right)} \int_{-1}^{1}\left(\frac{d^{k-1}}{d x^{k-1}}\left(1-x^{2}\right)^{k+\lambda-\frac{1}{2}}\right) B_{n-1}(x) d x=\cdots \\
& =\frac{(k+\lambda) \Gamma(\lambda)(-n)(-(n-1)) \cdots(-(n-k+1))}{(-2)^{k} \sqrt{\pi} \Gamma\left(k+\lambda+\frac{1}{2}\right)} \int_{-1}^{1}\left(1-x^{2}\right)^{k+\lambda-\frac{1}{2}} B_{n-k}(x) d x \\
& =\frac{(k+\lambda) \Gamma(\lambda)}{2^{k} \sqrt{\pi} \Gamma\left(k+\lambda+\frac{1}{2}\right)} \times \frac{n !}{(n-k) !} \int_{-1}^{1}\left(1-x^{2}\right)^{k+\lambda-\frac{1}{2}} B_{n-k}(x) d x .
\end{aligned}
$$

From (1.10) and (2.7), we can derive the following equation:

$$
\begin{aligned}
& \int_{-1}^{1}\left(1-x^{2}\right)^{k+\lambda-\frac{1}{2}} B_{n-k}(x) d x \\
& \quad=\sum_{l=0}^{n-k}\left(\begin{array}{c}
n-k \\
l
\end{array}\right) B_{n-k-l} \int_{-1}^{1}\left(1-x^{2}\right)^{k+\lambda-\frac{1}{2}} x^{l} d x \\
& \quad=\sum_{l=0}^{n-k}\left(\begin{array}{c}
n-k \\
l
\end{array}\right) B_{n-k-l}\left(1+(-1)^{l}\right) \int_{0}^{1}\left(1-x^{2}\right)^{k+\lambda-\frac{1}{2}} x^{l} d x .
\end{aligned}
$$


Let us consider that $l \equiv 0(\bmod 2)$. Then, by $(2.9)$, we get

$$
\begin{aligned}
& \int_{-1}^{1}\left(1-x^{2}\right)^{k+\lambda-\frac{1}{2}} B_{n-k}(x) d x \\
& =2 \sum_{0 \leq l \leq n-k, l \equiv 0(\bmod 2)}\left(\begin{array}{c}
n-k \\
l
\end{array}\right) B_{n-k-l} \int_{0}^{1}\left(1-x^{2}\right)^{k+\lambda-\frac{1}{2}} x^{l} d x \\
& =\sum_{0 \leq l \leq n-k, l \equiv 0(\bmod 2)}\left(\begin{array}{c}
n-k \\
l
\end{array}\right) B_{n-k-l} \int_{0}^{1}(1-y)^{k+\lambda-\frac{1}{2}} y^{\frac{l-1}{2}} d y \\
& =\sum_{0 \leq l \leq n-k, l \equiv 0(\bmod 2)}\left(\begin{array}{c}
n-k \\
l
\end{array}\right) B_{n-k-l} \frac{\Gamma\left(k+\lambda+\frac{1}{2}\right) \Gamma\left(\frac{l+1}{2}\right)}{\Gamma\left(\frac{2 k+2 \lambda+l+2}{2}\right)} .
\end{aligned}
$$

For $l \in \mathbb{Z}_{+}$with $l \equiv 0(\bmod 2)$, we have

$$
\begin{aligned}
\Gamma\left(\frac{l+1}{2}\right) & =\Gamma\left(\frac{l-1}{2}+1\right)=\frac{l-1}{2} \Gamma\left(\frac{l-1}{2}\right) \\
& =\left(\frac{l-1}{2}\right)\left(\frac{l-3}{2}\right) \Gamma\left(\frac{l-3}{2}\right)=\cdots \\
& =\left(\frac{l-1}{2}\right)\left(\frac{l-3}{2}\right) \cdots\left(\frac{1}{2}\right) \Gamma\left(\frac{1}{2}\right)=\frac{\left(\frac{1}{2}\right)^{l} l ! \Gamma\left(\frac{1}{2}\right)}{\left(\frac{l}{2}\right) !}=\frac{l ! \sqrt{\pi}}{2^{l}\left(\frac{l}{2}\right) !} .
\end{aligned}
$$

By (2.10) and (2.11), we get

$$
\begin{aligned}
& \int_{-1}^{1}\left(1-x^{2}\right)^{k+\lambda-\frac{1}{2}} B_{n-k}(x) d x \\
& =\sum_{0 \leq l \leq n-k, l \equiv 0(\bmod 2)}\left(\begin{array}{c}
n-k \\
l
\end{array}\right) B_{n-k-l} \frac{\Gamma\left(k+\lambda+\frac{1}{2}\right) \Gamma\left(\frac{l+1}{2}\right)}{\Gamma\left(\frac{2 k+2 \lambda+l+2}{2}\right)} \\
& =\sum_{0 \leq l \leq n-k, l \equiv 0(\bmod 2)}\left(\begin{array}{c}
n-k \\
l
\end{array}\right) B_{n-k-l} \frac{l ! \sqrt{\pi}}{2^{l}\left(\frac{l}{2}\right) !} \times \frac{\Gamma\left(k+\lambda+\frac{1}{2}\right)}{\Gamma\left(\frac{2 k+2 \lambda+l+2}{2}\right)} .
\end{aligned}
$$

From (2.8) and (2.12), we have

$$
d_{k}=\frac{n !(k+\lambda) \Gamma(\lambda)}{2^{k}(n-k) !} \sum_{0 \leq l \leq n-k, l \equiv 0(\bmod 2)} \frac{\left(\begin{array}{c}
n-k \\
l
\end{array}\right) B_{n-k-l} l !}{2^{l}\left(\frac{l}{2}\right) ! \Gamma\left(\frac{2 k+2 \lambda+l+2}{2}\right)} .
$$

Therefore, by (2.13) and Proposition 2.1, we obtain the following theorem.

Theorem 2.2 For $n \in \mathbb{Z}_{+}$, we have

$$
\frac{B_{n}(x)}{n !}=\Gamma(\lambda) \sum_{k=0}^{n}\left(\frac{(k+\lambda)}{2^{k}(n-k) !} \sum_{0 \leq l \leq n-k, l \equiv 0(\bmod 2)} \frac{\left(\begin{array}{c}
n-k \\
l
\end{array}\right) B_{n-k-l} l !}{2^{l}\left(\frac{l}{2}\right) ! \Gamma\left(\frac{2 k+2 \lambda+l+2}{2}\right)}\right) C_{k}^{(\lambda)}(x) .
$$

By the same method, we get

$$
\frac{E_{n}(x)}{n !}=\Gamma(\lambda) \sum_{k=0}^{n}\left(\frac{(k+\lambda)}{2^{k}(n-k) !} \sum_{0 \leq l \leq n-k, l \equiv 0(\bmod 2)} \frac{\left(\begin{array}{c}
n-k \\
l
\end{array}\right) E_{n-k-l} l !}{2^{l}\left(\frac{l}{2}\right) ! \Gamma\left(\frac{2 k+2 \lambda+l+2}{2}\right)}\right) C_{k}^{(\lambda)}(x) .
$$


From (1.1), we note that

$$
\begin{aligned}
C_{n-k}^{(\lambda)}(x) C_{k}^{(\lambda)}(x) \\
=\left(\begin{array}{c}
n-k+2 \lambda-1 \\
n-k
\end{array}\right) \sum_{l=0}^{n-k} \frac{\left(\begin{array}{c}
n-k \\
l
\end{array}\right)(2 \lambda+n-k)_{l}}{\left(\lambda+\frac{1}{2}\right)_{l}}\left(\frac{x-1}{2}\right)^{l}\left(\begin{array}{c}
k+2 \lambda-1 \\
k
\end{array}\right) \\
\quad \times \sum_{m-0}^{k} \frac{\left(\begin{array}{c}
k \\
m
\end{array}\right)(2 \lambda+k)_{m}}{\left(\lambda+\frac{1}{2}\right)_{m}}\left(\frac{x-1}{2}\right)^{m} \\
=\left(\begin{array}{c}
n-k+2 \lambda-1 \\
n-k
\end{array}\right)\left(\begin{array}{c}
k+2 \lambda-1 \\
k
\end{array}\right) \\
\quad \times \sum_{p=0}^{n}\left(\sum_{m=0}^{p} \frac{\left(\begin{array}{c}
n-k \\
p-m
\end{array}\right)\left(\begin{array}{l}
k \\
m
\end{array}\right)(2 \lambda+k)_{m}(2 \lambda+n-k)_{p-m}}{\left(\lambda+\frac{1}{2}\right)_{m}\left(\lambda+\frac{1}{2}\right)_{p-m}}\right)\left(\frac{x-1}{2}\right)^{p} .
\end{aligned}
$$

Let us take $p(x)=C_{k}^{(\lambda)}(x) C_{n-k}^{(\lambda)}(x) \in \mathbb{P}_{n}$. From Proposition 2.1, $p(x)$ can be rewritten as

$$
p(x)=C_{k}^{(\lambda)}(x) C_{n-k}^{(\lambda)}(x)=\sum_{r=0}^{n} d_{r} C_{r}^{(\lambda)}(x) \quad\left(d_{r} \in \mathbb{R}\right) .
$$

Then, by Proposition 2.1 and (2.15), we get

$$
\begin{aligned}
d_{r}= & \frac{(r+\lambda) \Gamma(\lambda)}{(-2)^{r} \sqrt{\pi} \Gamma\left(r+\lambda+\frac{1}{2}\right)} \int_{-1}^{1}\left(\frac{d^{r}}{d x^{r}}\left(1-x^{2}\right)^{r+\lambda-\frac{1}{2}}\right) C_{k}^{(\lambda)}(x) C_{n-k}^{(\lambda)}(x) d x \\
= & \frac{(r+\lambda) \Gamma(\lambda)}{(-2)^{r} \sqrt{\pi} \Gamma\left(r+\lambda+\frac{1}{2}\right)}\left(\begin{array}{c}
n-k+2 \lambda-1 \\
n-k
\end{array}\right)\left(\begin{array}{c}
k+2 \lambda-1 \\
k
\end{array}\right) \\
& \times \sum_{p=0}^{n}\left(\sum_{m=0}^{p} \frac{\left(\begin{array}{c}
n-k \\
p-m
\end{array}\right)\left(\begin{array}{c}
k \\
m
\end{array}\right)(2 \lambda+k)_{m}}{\left(\lambda+\frac{1}{2}\right)_{m}\left(\lambda+\frac{1}{2}\right)_{p-m}}(2 \lambda+n-k)_{p-m}\right) \\
& \times \int_{-1}^{1}\left(\frac{d^{r}}{d x^{r}}\left(1-x^{2}\right)^{r+\lambda-\frac{1}{2}}\right)\left(\frac{x-1}{2}\right)^{p} d x \\
& \frac{(r+\lambda) \Gamma(\lambda)}{(-2)^{r} \sqrt{\pi} \Gamma\left(r+\lambda+\frac{1}{2}\right)}\left(\begin{array}{c}
n-k+2 \lambda-1 \\
n-k
\end{array}\right)\left(\begin{array}{c}
k+2 \lambda-1 \\
k
\end{array}\right) \\
& \times \sum_{p=r}^{n}\left(\sum_{m=0}^{p} \frac{\left(\begin{array}{c}
n-k \\
p-m
\end{array}\right)\left(\begin{array}{c}
k \\
m
\end{array}\right)(2 \lambda+k)_{m}}{\left(\lambda+\frac{1}{2}\right)_{m}\left(\lambda+\frac{1}{2}\right)_{p-m}}(2 \lambda+n-k)_{p-m}\right) \\
& \times \int_{-1}^{1}\left(\frac{d^{r}}{d x^{r}}\left(1-x^{2}\right)^{r+\lambda-1}\right)\left(\frac{x-1}{2}\right)^{p} d x .
\end{aligned}
$$

It is not difficult to show that

$$
\begin{aligned}
& \int_{-1}^{1}\left(\frac{d^{r}}{d x^{r}}\left(1-x^{2}\right)^{r+\lambda-\frac{1}{2}}\right)\left(\frac{x-1}{2}\right)^{p} d x \\
& \quad=\frac{(-1)^{r} p !}{2^{p}(p-r) !} \int_{-1}^{1}\left(1-x^{2}\right)^{r+\lambda-\frac{1}{2}}(1-x)^{p-r}(-1)^{p-r} d x \\
& =\frac{(-1)^{p} p !}{2^{p}(p-r) !} \int_{-1}^{1}(1-x)^{p+\lambda-\frac{1}{2}}(1+x)^{r+\lambda-\frac{1}{2}} d x
\end{aligned}
$$




$$
\begin{aligned}
& =\frac{(-1)^{p} p !}{2^{p}(p-r) !} \int_{0}^{1}(2-2 y)^{p+\lambda-\frac{1}{2}}(2 y)^{r+\lambda-\frac{1}{2}} 2 d y \\
& =\frac{(-1)^{p} 2^{p+\lambda-\frac{1}{2}+r+\lambda-\frac{1}{2}+1}}{2^{p}} \times \frac{p !}{(p-r) !} \int_{0}^{1}(1-y)^{p+\lambda-\frac{1}{2}} y^{r+\lambda-\frac{1}{2}} d y \\
& =(-1)^{p} 2^{r+2 \lambda} \frac{p !}{(p-r) !} \times \frac{\Gamma\left(p+\lambda+\frac{1}{2}\right) \Gamma\left(r+\lambda+\frac{1}{2}\right)}{\Gamma(r+p+2 \lambda+1)} .
\end{aligned}
$$

From the fundamental theorem of gamma function, we have

$$
\frac{\Gamma\left(p+\lambda+\frac{1}{2}\right)}{\Gamma(r+p+2 \lambda+1)}=\frac{\left(p+\lambda-\frac{1}{2}\right) \cdots\left(\lambda+\frac{1}{2}\right) \Gamma\left(\lambda+\frac{1}{2}\right)}{(r+p+2 \lambda) \cdots 2 \lambda \Gamma(2 \lambda)}=\frac{\left(\lambda+\frac{1}{2}\right)_{p} \sqrt{\pi} 2^{1-\lambda}}{(2 \lambda)_{r+p+1} \Gamma(\lambda)} .
$$

By (2.18) and (2.19), we get

$$
\begin{aligned}
& \int_{-1}^{1}\left(\frac{d^{r}}{d x^{r}}\left(1-x^{2}\right)^{r+\lambda-\frac{1}{2}}\right)\left(\frac{x-1}{2}\right)^{p} d x \\
& =(-1)^{p} 2^{r+2 \lambda} \frac{p !}{(p-r) !} \times \frac{\Gamma\left(p+\lambda+\frac{1}{2}\right) \Gamma\left(r+\lambda+\frac{1}{2}\right)}{\Gamma(r+p+2 \lambda+1)} \\
& \quad=(-1)^{p} 2^{r+2 \lambda} \frac{p !}{(p-r) !} \Gamma\left(r+\lambda+\frac{1}{2}\right) \times \frac{\left(\lambda+\frac{1}{2}\right)_{p} \sqrt{\pi} 2^{1-\lambda}}{(2 \lambda)_{r+p+1} \Gamma(\lambda)} \\
& =(-1)^{p} 2^{r+\lambda+1} \frac{p !}{(p-r) !} \Gamma\left(r+\lambda+\frac{1}{2}\right) \times \frac{\left(\lambda+\frac{1}{2}\right)_{p} \sqrt{\pi}}{(2 \lambda)_{r+p+1} \Gamma(\lambda)} .
\end{aligned}
$$

From (2.17) and (2.20), we have

$$
\begin{aligned}
d_{r}= & \frac{(r+\lambda) \Gamma(\lambda)}{(-2)^{r} \sqrt{\pi} \Gamma\left(r+\lambda+\frac{1}{2}\right)}\left(\begin{array}{c}
n-k+2 \lambda-1 \\
n-k
\end{array}\right)\left(\begin{array}{c}
k+2 \lambda-1 \\
k
\end{array}\right) \\
& \times \sum_{p=r}^{n}\left(\sum_{m=0}^{p} \frac{\left(\begin{array}{c}
n-k \\
p-m
\end{array}\right)\left(\begin{array}{l}
k \\
m
\end{array}\right)(2 \lambda+k)_{m}}{\left(\lambda+\frac{1}{2}\right)_{m}\left(\lambda+\frac{1}{2}\right)_{p-m}}(2 \lambda+n-k)_{p-m}\right) \\
& \times(-1)^{p} 2^{r+\lambda+1} \frac{p !}{(p-r) !} \Gamma\left(\lambda+\frac{1}{2}+r\right) \times \frac{\left(\lambda+\frac{1}{2}\right)_{p} \sqrt{\pi}}{(2 \lambda)_{r+p+1} \Gamma(\lambda)} \\
= & (-1)^{r+p} 2^{\lambda+1}(r+\lambda)\left(\begin{array}{c}
n-k+2 \lambda-1 \\
n-k
\end{array}\right)\left(\begin{array}{c}
k+2 \lambda-1 \\
k
\end{array}\right) \\
& \times \sum_{p=r}^{n}\left(\sum_{m=0}^{p} \frac{\left(\begin{array}{l}
n-k \\
p-m
\end{array}\right)\left(\begin{array}{l}
k \\
m
\end{array}\right)(2 \lambda+k)_{m}}{\left(\lambda+\frac{1}{2}\right)_{m}\left(\lambda+\frac{1}{2}\right)_{p-m}}(2 \lambda+n-k)_{p-m} \frac{p !\left(\lambda+\frac{1}{2}\right)_{p}}{(p-r) !(2 \lambda)_{r+p+1}}\right) .
\end{aligned}
$$

Therefore, by (2.21), we obtain the following theorem.

Theorem 2.3 For $n, k \in \mathbb{Z}_{+}$with $n \geq k$, we have

$$
\begin{aligned}
C_{n-k}^{(\lambda)}(x) C_{k}^{(\lambda)}(x)= & 2^{\lambda+1}\left(\begin{array}{c}
n-k+2 \lambda-1 \\
n-k
\end{array}\right)\left(\begin{array}{c}
k+2 \lambda-1 \\
k
\end{array}\right) \sum_{r=0}^{n} \sum_{p=r}^{n} \sum_{m=0}^{p}\left\{(r+\lambda)(-1)^{p+r}\right. \\
& \left.\times \frac{\left(\begin{array}{c}
n-k \\
p-m
\end{array}\right)\left(\begin{array}{l}
k \\
m
\end{array}\right)(2 \lambda+k)^{m}(2 \lambda+n-k)_{p-m} p !\left(\lambda+\frac{1}{2}\right)_{p}}{\left(\lambda+\frac{1}{2}\right)_{m}\left(\lambda+\frac{1}{2}\right)_{p-m}(p-r) !(2 \lambda)_{r+p+1}}\right\} C_{r}^{(\lambda)}(x) .
\end{aligned}
$$


Let us take $p(x)=C_{n}^{(\lambda)}(x) \in \mathbb{P}_{n}$. Then, from (1.1), we have

$$
\begin{aligned}
C_{n}^{(\lambda)}(x) & =\frac{\Gamma\left(\lambda+\frac{1}{2}\right) \Gamma(n+2 \lambda)}{\Gamma(2 \lambda) \Gamma\left(n+\lambda+\frac{1}{2}\right)} P_{n}^{\left(\lambda-\frac{1}{2}, \lambda-\frac{1}{2}\right)}(x) \\
& =\frac{(n+2 \lambda-1) \cdots(2 \lambda)}{\left(n+\lambda-\frac{1}{2}\right) \cdots\left(\lambda+\frac{1}{2}\right)} P_{n}^{\left(\lambda-\frac{1}{2}, \lambda-\frac{1}{2}\right)}(x)=\frac{\left(\begin{array}{c}
n+2 \lambda-1 \\
n
\end{array}\right)}{\left(\begin{array}{c}
n+\lambda-\frac{1}{2} \\
n
\end{array}\right)} P_{n}^{\left(\lambda-\frac{1}{2}, \lambda-\frac{1}{2}\right)}(x) .
\end{aligned}
$$

In the previous paper, we have shown that

$$
P_{n}^{(\alpha, \beta)}(x)=\sum_{k=0}^{n}\left(\begin{array}{c}
n+\alpha \\
n-k
\end{array}\right)\left(\begin{array}{c}
n+\beta \\
k
\end{array}\right)\left(\frac{x-1}{2}\right)^{k}\left(\frac{x+1}{2}\right)^{n-k} \quad(\text { see }[14]) .
$$

From (2.22) and (2.23), we have

$$
C_{n}^{(\lambda)}(x)=\frac{\left(\begin{array}{c}
n+2 \lambda-1 \\
n
\end{array}\right)}{\left(\begin{array}{c}
n+\lambda-\frac{1}{2} \\
n
\end{array}\right)} \sum_{k=0}^{n}\left(\begin{array}{c}
n+\lambda-\frac{1}{2} \\
n-k
\end{array}\right)\left(\begin{array}{c}
n+\lambda-\frac{1}{2} \\
k
\end{array}\right)\left(\frac{x-1}{2}\right)^{k}\left(\frac{x+1}{2}\right)^{n-k}
$$

and

$$
\frac{d^{k}}{d x^{k}} C_{n}^{(\lambda)}(x)=2^{k} \lambda^{k} C_{n-k}^{(\lambda+k)}(x)
$$

Let $p(x)=C_{n}^{(\lambda)}(x)=\sum_{k=0}^{n} d_{k} C_{k}^{(\lambda)}(x)$. Then, by Proposition 2.1, we get

$$
\begin{aligned}
d_{k} & =\frac{(k+\lambda) \Gamma(\lambda)}{(-2)^{k} \sqrt{\pi} \Gamma\left(k+\lambda+\frac{1}{2}\right)} \int_{-1}^{1}\left(\frac{d^{k}}{d x^{k}}\left(1-x^{2}\right)^{k+\lambda-\frac{1}{2}}\right) C_{n}^{(\lambda)}(x) d x \\
& =\frac{(k+\lambda) \Gamma(\lambda)}{(-2)^{k} \sqrt{\pi} \Gamma\left(k+\lambda+\frac{1}{2}\right)}(-1)^{k} 2^{k} \lambda^{k} \int_{-1}^{1}\left(1-x^{2}\right)^{k+\lambda-\frac{1}{2}} C_{n-k}^{(\lambda+k)}(x) d x \\
& =\frac{\lambda^{k}(k+\lambda) \Gamma(\lambda)}{\sqrt{\pi} \Gamma\left(k+\lambda+\frac{1}{2}\right)} \int_{-1}^{1}\left(1-x^{2}\right)^{k+\lambda-\frac{1}{2}} C_{n-k}^{(\lambda+k)}(x) d x .
\end{aligned}
$$

By (2.24), we get

$$
\begin{aligned}
& C_{n-k}^{(\lambda+k)}(x) \\
& \quad=\frac{\left(\begin{array}{c}
n-k+2(\lambda+k)-1 \\
n-k
\end{array}\right)}{\left(\begin{array}{c}
n-k+\lambda+k-\frac{1}{2} \\
n-k
\end{array}\right)} \sum_{l=0}^{n-k}\left(\begin{array}{c}
n-k+\lambda+k-\frac{1}{2} \\
n-k-l
\end{array}\right)\left(\begin{array}{c}
n-k+\lambda+k-\frac{1}{2} \\
l
\end{array}\right)\left(\frac{x-1}{2}\right)^{l}\left(\frac{x+1}{2}\right)^{n-k-l} \\
& \quad=\frac{\left(\begin{array}{c}
n+k+2 \lambda-1 \\
n-k
\end{array}\right)}{\left(\begin{array}{c}
n+\lambda-\frac{1}{2} \\
n-k
\end{array}\right)} \sum_{l=0}^{n-k}\left(\begin{array}{c}
n+\lambda-\frac{1}{2} \\
n-k-l
\end{array}\right)\left(\begin{array}{c}
n+\lambda-\frac{1}{2} \\
l
\end{array}\right)\left(\frac{x-1}{2}\right)^{l}\left(\frac{x+1}{2}\right)^{n-k-l}
\end{aligned}
$$

From (2.26) and (2.27), we have

$$
\begin{aligned}
d_{k}= & \frac{\lambda^{k}(k+\lambda) \Gamma(\lambda)}{\sqrt{\pi} \Gamma\left(k+\lambda+\frac{1}{2}\right)} \times \frac{\left(\begin{array}{c}
n+k+2 \lambda-1 \\
n-k
\end{array}\right)}{\left(\begin{array}{c}
n+\lambda-\frac{1}{2} \\
n-k
\end{array}\right)} \sum_{l=0}^{n-k}\left(\begin{array}{c}
n+\lambda-\frac{1}{2} \\
n-k-l
\end{array}\right)\left(\begin{array}{c}
n+\lambda-\frac{1}{2} \\
l
\end{array}\right)(-1)^{l}\left(\frac{1}{2}\right)^{n-k} \\
& \times \int_{-1}^{1}(1-x)^{k+\lambda-\frac{1}{2}+l}(1+x)^{\lambda+n-\frac{1}{2}-l} d x .
\end{aligned}
$$


It is easy to show that

$$
\begin{aligned}
& \int_{-1}^{1}(1-x)^{k+\lambda-\frac{1}{2}+l}(1+x)^{\lambda+n-l-\frac{1}{2}} d x \\
& \quad=\int_{0}^{1}(2-2 y)^{k+\lambda-\frac{1}{2}+l}(2 y)^{\lambda+n-l-\frac{1}{2}} 2 d y \\
& =2^{n+2 \lambda+k} \int_{0}^{1}(1-y)^{k+\lambda+l-\frac{1}{2}} y^{\lambda+n-l-\frac{1}{2}} d y \\
& =2^{k+n+2 \lambda} \frac{\Gamma\left(k+\lambda+l+\frac{1}{2}\right) \Gamma\left(\lambda+n-l+\frac{1}{2}\right)}{\Gamma(k+n+2 \lambda)} .
\end{aligned}
$$

By the fundamental theorem of gamma function, we see that

$$
\begin{aligned}
& \Gamma\left(k+\lambda+l+\frac{1}{2}\right)=\left(\begin{array}{c}
k+\lambda+l-\frac{1}{2} \\
l
\end{array}\right) l ! \Gamma\left(k+\lambda+\frac{1}{2}\right), \\
& \Gamma\left(\lambda+n-l+\frac{1}{2}\right)=\left(\begin{array}{c}
\lambda+n-l-\frac{1}{2} \\
n-l
\end{array}\right)(n-l) ! \Gamma\left(\lambda+\frac{1}{2}\right),
\end{aligned}
$$

and

$$
\Gamma(k+2 \lambda+n)=\left(\begin{array}{c}
k+2 \lambda+n-1 \\
n+k
\end{array}\right)(n+k) ! \Gamma(2 \lambda) .
$$

As is well known, the duplication formula for the gamma function is given by

$$
\Gamma(z) \Gamma\left(z+\frac{1}{2}\right)=2^{1-2 z} \sqrt{\pi} \Gamma(2 z)
$$

By (2.29), (2.30), (2.31), and (2.32), we get

$$
\int_{-1}^{1}(1-x)^{k+\lambda+l-\frac{1}{2}}(1+x)^{\lambda+n-l-\frac{1}{2}} d x=2^{k+n+1} \frac{\left(\begin{array}{c}
k+\lambda+l-\frac{1}{2} \\
l
\end{array}\right)\left(\begin{array}{c}
\lambda+n-l-\frac{1}{2} \\
n-l
\end{array}\right) \Gamma\left(k+\lambda+\frac{1}{2}\right)}{\left(\begin{array}{c}
n \\
l
\end{array}\right)\left(\begin{array}{c}
k+2 \lambda+n-1 \\
n+k
\end{array}\right)\left(\begin{array}{c}
n+k \\
k
\end{array}\right) k ! \Gamma(\lambda)} \sqrt{\pi} .
$$

From (2.28) and (2.34), we have

$$
\begin{aligned}
d_{k}= & \lambda^{k}(k+\lambda) 2^{2 k+1} \frac{\left(\begin{array}{c}
n+k+2 \lambda-1 \\
n-k
\end{array}\right)}{\left(\begin{array}{c}
n+\lambda-\frac{1}{2} \\
n-k
\end{array}\right)} \\
& \times \sum_{l=0}^{n-k}\left(\begin{array}{c}
n+\lambda-\frac{1}{2} \\
n-k-l
\end{array}\right)\left(\begin{array}{c}
n+\lambda-\frac{1}{2} \\
l
\end{array}\right)(-1)^{l} \frac{\left(\begin{array}{c}
k+\lambda+l-\frac{1}{2} \\
l
\end{array}\right)\left(\begin{array}{c}
\lambda+n-l-\frac{1}{2} \\
n-l
\end{array}\right)}{\left(\begin{array}{c}
n \\
l
\end{array}\right)\left(\begin{array}{c}
k+2 \lambda+n-1 \\
n+k
\end{array}\right)\left(\begin{array}{c}
n+k \\
k
\end{array}\right) k !} .
\end{aligned}
$$

Therefore, by (2.35), we obtain the following theorem.

Theorem 2.4 For $n \in \mathbb{Z}_{+}$, we have

$$
\begin{aligned}
C_{n}^{(\lambda)}(x)= & \sum_{k=0}^{n}\left\{\frac{\lambda^{k}(k+\lambda) 2^{2 k+1}\left(\begin{array}{c}
n+k+2 \lambda-1 \\
n-k
\end{array}\right)}{\left(\begin{array}{c}
n+\lambda-\frac{1}{2} \\
n-k
\end{array}\right)}\right. \\
& \left.\times \sum_{l=0}^{n-k} \frac{\left(\begin{array}{c}
n+\lambda-\frac{1}{2} \\
n-k-l
\end{array}\right)\left(\begin{array}{c}
n+\lambda-\frac{1}{2} \\
l
\end{array}\right)(-1)^{l}\left(\begin{array}{c}
k+\lambda+l-\frac{1}{2} \\
l
\end{array}\right)}{\left(\begin{array}{c}
n \\
l
\end{array}\right)\left(\begin{array}{c}
k+2 \lambda+n-1 \\
n+k
\end{array}\right)\left(\begin{array}{c}
n+k \\
k
\end{array}\right) k !}\left(\begin{array}{c}
\lambda+l-\frac{1}{2} \\
n-l
\end{array}\right)\right\} C_{k}^{(\lambda)}(x) .
\end{aligned}
$$




\section{Competing interests}

The authors declare that they have no competing interests.

\section{Authors' contributions}

All authors contributed equally to the manuscript and typed, read, and approved the final manuscript.

\section{Author details}

'Department of Mathematics, Sogang University, Seoul, 121-742, Republic of Korea. ${ }^{2}$ Department of Mathematics, Kwangwoon University, Seoul, 139-701, Republic of Korea. ${ }^{3}$ Department of Mathematics Education, Kyungpook National University, Taegu, 702-701, Republic of Korea.

\section{Acknowledgements}

This research was supported by the Basic Science Research Program through the National Research Foundation of Korea (NRF) funded by the Ministry of Education, Science and Technology 2012R1A1A2003786.

Received: 13 August 2012 Accepted: 30 November 2012 Published: 19 December 2012

\section{References}

1. Shah, M: Some properties associated with generalized integral transform in two variables. Univ. Brasov Lucrari Sti. 18, 15-27 (1976)

2. de Vicente, Jl, Gandy, S, Sánchez-Ruiz, J: Information entropy of Gegenbauer polynomials of integer parameter. J. Phys. A, Math. Theor. 40, 8345-8361 (2007)

3. Al-Salam, WA, Carlitz, L: The Gegenbauer addition theorem. J. Math. Phys. 42, 147-156 (1963)

4. McFadden, JA: A diagonal expansion in Gegenbauer polynomials for a class of second-order probability densities. SIAM J. Appl. Math. 14, 1433-1436 (1966)

5. Bayad, A, Kim, T: Identities involving values of Bernstein, $q$-Bernoulli, and $q$-Euler polynomials. Russ. J. Math. Phys. 18(2), 133-143 (2011)

6. Bayad, A: Modular properties of elliptic Bernoulli and Euler functions. Adv. Stud. Contemp. Math. 20(3), 389-401 (2010)

7. Buyarov, VS, Lopez-Artes, P, Martinez-Finkelshtein, A, Van Assche, W: Information entropy of Gegenbauer polynomials. J. Phys. A, Math. Gen. 33, 6549-6560 (2000)

8. Cangul, IN, Kurt, V, Ozden, H, Simsek, Y: On the higher-order w- $q$-Genocchi numbers. Adv. Stud. Contemp. Math. 19(1), 39-57 (2009)

9. Choi, J, Kim, DS, Kim, T, Kim, YH: Some arithmetic identities on Bernoulli and Euler numbers arising from the $p$-adic integrals on $\mathbb{Z}_{p}$. Adv. Stud. Contemp. Math. 22(2), 239-247 (2012)

10. Khan, S, Al-Gonah, AA, Yasmin, G: Generalized and mixed type Gegenbauer polynomials. J. Math. Anal. Appl. 390(1), 197-207 (2012)

11. Kim, T, Choi, J, Kim, YH, Ryoo, CS: On q-Bernstein and q-Hermite polynomials. Proc. Jangjeon Math. Soc. 14(2), $215-221$ (2011)

12. Kim, T: Some identities on the $q$-Euler polynomials of higher order and $q$-Stirling numbers by the fermionic $p$-adic integral on $\mathbb{Z}_{p}$. Russ. J. Math. Phys. 16(4), 484-491 (2009)

13. Kim, T: Symmetry of power sum polynomials and multivariate fermionic $p$-adic invariant integral on $\mathbb{Z}_{p}$. Russ. J. Math Phys. 16(1), 93-96 (2009)

14. Kim, DS, Kim, T, Dolgy, DV: Some identities on Bernoulli and Hermite polynomials associated with Jacobi polynomials. Discrete Dyn. Nat. Soc. 2012, Article ID 584643 (2012)

15. Ozden, H, Cangul, IN, Simsek, Y: Remarks on q-Bernoulli numbers associated with Daehee numbers. Adv. Stud. Contemp. Math. 18(1), 41-48 (2009)

16. Rim, S-H, Lee, S-J: Some identities on the twisted $(h, q)$-Genocchi numbers and polynomials associated with q-Bernstein polynomials. Int. J. Math. Math. Sci. 2011, Article ID 482840 (2011)

17. Ryoo, CS: Some relations between twisted $q$-Euler numbers and Bernstein polynomials. Adv. Stud. Contemp. Math. 21(2), 217-223 (2011)

18. Sanchez-Ruiz, J: Information entropy of Gegenbauer polynomials and Gaussian quadrature. J. Phys. A, Math. Gen. 36, 4857-4865 (2003)

19. Simsek, Y: Special functions related to Dedekind-type DC-sums and their applications. Russ. J. Math. Phys. 17(4), 495-508 (2010)

20. Simsek, Y: Generating functions of the twisted Bernoulli numbers and polynomials associated with their interpolation functions. Adv. Stud. Contemp. Math. 16(2), 251-278 (2008)

21. Sneddon, IN: The evaluation of an integral involving the product of two Gegenbauer polynomials. SIAM Rev. 9 569-572 (1967)

22. Shah, M: Applications of Gegenbauer (ultraspherical) polynomials in cooling of a heated cylinder. An. Univ. Timisoara, Ser. Sti. Mat. 8, 207-212 (1970)

doi:10.1186/1687-1847-2012-219

Cite this article as: Kim et al.: Some identities involving Gegenbauer polynomials. Advances in Difference Equations 2012 2012:219. 